\title{
Endpoint Boundedness of Riesz Transforms on Hardy Spaces Associated with Operators
}

\author{
Jun Cao · Dachun Yang · Sibei Yang
}

\begin{abstract}
Let $L_{1}$ be a nonnegative self-adjoint operator in $L^{2}\left(\mathbb{R}^{n}\right)$ satisfying the DaviesGaffney estimates and $L_{2}$ a second order divergence form elliptic operator with complex bounded measurable coefficients. A typical example of $L_{1}$ is the Schrödinger operator $-\Delta+V$, where $\Delta$ is the Laplace operator on $\mathbb{R}^{n}$ and $0 \leq V \in L_{\text {loc }}^{1}\left(\mathbb{R}^{n}\right)$. Let $H_{L_{i}}^{p}\left(\mathbb{R}^{n}\right)$ be the Hardy space associated to $L_{i}$ for $i \in\{1,2\}$. In this paper, the authors prove that the Riesz transform $D\left(L_{i}^{-1 / 2}\right)$ is bounded from $H_{L_{i}}^{p}\left(\mathbb{R}^{n}\right)$ to the classical weak Hardy space $W H^{p}\left(\mathbb{R}^{n}\right)$ in the critical case that $p=n /(n+1)$. Recall that it is known that $D\left(L_{i}^{-1 / 2}\right)$ is bounded from $H_{L_{i}}^{p}\left(\mathbb{R}^{n}\right)$ to the classical Hardy space $H^{p}\left(\mathbb{R}^{n}\right)$ when $p \in(n /(n+1), 1]$.

Keywords Riesz transform · Davies-Gaffney estimate · Schrödinger operator · Second order elliptic operator · Hardy space · Weak Hardy space

Mathematics Subject Classification (2010) Primary 47B06 - Secondary 42B20 . 42B25 - 42B30 - 35J10
\end{abstract}

\section{Introduction}

The Hardy spaces, as a suitable substitute of Lebesgue spaces $L^{p}\left(\mathbb{R}^{n}\right)$ when $p \in(0,1]$, play an important role in various fields of analysis and partial differential equations. For example, when $p \in(0,1]$, the Riesz transform $\nabla(-\Delta)^{-1 / 2}$ is not bounded on $L^{p}\left(\mathbb{R}^{n}\right)$, but bounded on the Hardy space $H^{p}\left(\mathbb{R}^{n}\right)$, where $\Delta$ is the Laplacian operator $\sum_{i=1}^{n} \frac{\partial^{2}}{\partial x_{i}^{2}}$ and $\nabla$ is the gradient operator $\left(\frac{\partial}{\partial x_{1}}, \cdots, \frac{\partial}{\partial x_{n}}\right)$ on $\mathbb{R}^{n}$. It is well known that the classical Hardy spaces $H^{p}\left(\mathbb{R}^{n}\right)$ are essentially related to $\Delta$, which has been intensively studied in, for example, $[7,14,30,32,33]$ and their references.

In recent years, the study of Hardy spaces associated to differential operators inspires great interests; see, for example, $[2,3,4,11,12,13,16,18,19,20,9]$ and their references.

J. Cao · D.-C. Yang (凶) · S.-B. Yang

School of Mathematical Sciences, Beijing Normal University, Laboratory of Mathematics and Complex Systems, Ministry of Education, Beijing 100875, People's Republic of China

e-mails: dcyang@bnu.edu.cn

J. Cao

e-mails: caojun1860@mail.bnu.edu.cn

S.-B. Yang

e-mails: yangsibei@mail.bnu.edu.cn 
In particular, Auscher, Duong and McIntosh [2] first introduced the Hardy space $H_{L}^{1}\left(\mathbb{R}^{n}\right)$ associated to $L$, where the heat kernel generated by $L$ satisfies a pointwise Poisson type upper bound. Later, Duong and Yan $[10,11]$ introduced its dual space $\mathrm{BMO}_{L}\left(\mathbb{R}^{n}\right)$ and established the dual relation between $H_{L}^{1}\left(\mathbb{R}^{n}\right)$ and $\mathrm{BMO}_{L^{*}}\left(\mathbb{R}^{n}\right)$, where $L^{*}$ denotes the adjoint operator of $L$ in $L^{2}\left(\mathbb{R}^{n}\right)$. Yan [35] further introduced the Hardy space $H_{L}^{p}\left(\mathbb{R}^{n}\right)$ for some $p \in(0,1]$ but near to 1 and generalized these results to $H_{L}^{p}\left(\mathbb{R}^{n}\right)$ and their dual spaces. A theory of the Orlicz-Hardy space and its dual space associated to a such $L$ were developed in $[25,22]$.

Moreover, for the Schrödinger operator $-\Delta+V$, Dziubański and Zienkiewicz [12, 13] first introduced the Hardy spaces $H_{-\Delta+V}^{p}\left(\mathbb{R}^{n}\right)$ with the nonnegative potential $V$ belonging to the reverse Hölder class $B_{q}\left(\mathbb{R}^{n}\right)$ for certain $q \in(1, \infty)$. As a special case, the Hardy space $H_{-\Delta+V}^{p}\left(\mathbb{R}^{n}\right)$ associated with $-\Delta+V$ with $0 \leq V \in L_{\mathrm{loc}}^{1}\left(\mathbb{R}^{n}\right)$ and $p \in(0,1]$ but near to 1 was also studied in, for example, $[11,16,35,25,36,37,21,8]$. More generally, for nonnegative self-adjoint operators $L$ satisfying the Davies-Gaffney estimates, Hofmann et al. [16] introduced a new Hardy space $H_{L}^{1}\left(\mathbb{R}^{n}\right)$. In particular, when $L \equiv-\Delta+V$ with $0 \leq V \in L_{\text {loc }}^{1}\left(\mathbb{R}^{n}\right)$, Hofmann et al. originally showed that the Riesz transform $\nabla\left(L^{-1 / 2}\right)$ is bounded from $H_{L}^{1}\left(\mathbb{R}^{n}\right)$ to the classical Hardy space $H^{1}\left(\mathbb{R}^{n}\right)$. These results in [16] were further extended to the Orlicz-Hardy space and its dual space in [21]. In particular, as a special case of $[21$, Theorem 6.3$]$, it was proved that $\nabla(-\Delta+V)^{-1 / 2}$ with $0 \leq V \in L_{\text {loc }}^{1}\left(\mathbb{R}^{n}\right)$ is bounded from the Hardy space $H_{-\Delta+V}^{p}\left(\mathbb{R}^{n}\right)$ to $H^{p}\left(\mathbb{R}^{n}\right)$ if $p \in\left(\frac{n}{n+1}, 1\right]$.

Also, Auscher and Russ [4] studied the Hardy space $H_{L}^{1}$ on strongly Lipschitz domains associated with a second order divergence form elliptic operator $L$ whose heat kernels have the Gaussian upper bounds and certain regularity. Hofmann and Mayboroda [18, 19] and Hofmann et al. [20] introduced the Hardy and Sobolev spaces associated to a second order divergence form elliptic operator $L$ on $\mathbb{R}^{n}$ with complex bounded measurable coefficients. Notice that, for the second order divergence form elliptic operator $L$, the kernel of the heat semigroup may fail to satisfy the Gaussian upper bound estimate and, moreover, $L$ may not be nonnegative self-adjoint in $L^{2}\left(\mathbb{R}^{n}\right)$. Hofmann et al. [20] also proved that the associated Riesz transform $\nabla L^{-1 / 2}$ is bounded from $H_{L}^{p}\left(\mathbb{R}^{n}\right)$ to the classical Hardy space $H^{p}\left(\mathbb{R}^{n}\right)$ with $p \in\left(\frac{n}{n+1}, 1\right]$, which was also independently obtained by Jiang and Yang in [23, Theorem 7.4]. Moreover, a theory of the Orlicz-Hardy space and its dual space associated to $L$ were developed in $[23,24]$.

Recently, the Hardy space $H_{(-\Delta)^{2}+V^{2}}^{1}\left(\mathbb{R}^{n}\right)$ associated to the Schrödinger-type operators $(-\Delta)^{2}+V^{2}$ with $0 \leq V$ satisfying the reverse Hölder inequality was also studied in [5]. Moreover, the Hardy space $H_{L}^{p}\left(\mathbb{R}^{n}\right)$ associated to a one-to-one operator of type $\omega$ satisfying the $k$-Davies-Gaffney estimate and having a bounded $H_{\infty}$ functional calculus was introduced in [6], where $k \in \mathbb{N}$. Notice that when $k=1$, the $k$-Davies-Gaffney estimate is just the Davies-Gaffney estimate. Typical examples of such operators include the $2 k$ order divergence form homogeneous elliptic operator $T_{1}$ with complex bounded measurable coefficients and the $2 k$-order Schrödinger-type operator $T_{2} \equiv(-\Delta)^{k}+V^{k}$, where $0 \leq V \in$ $L_{\text {loc }}^{k}\left(\mathbb{R}^{n}\right)$. It was further proved that the associated Riesz transform $\nabla^{k} T_{i}^{-1 / 2}$ for $i \in\{1,2\}$ is bounded from $H_{T_{i}}^{p}\left(\mathbb{R}^{n}\right)$ to $H^{p}\left(\mathbb{R}^{n}\right)$ with $p \in\left(\frac{n}{n+k}, 1\right]$ in [6].

On the other hand, the weak Hardy space $W H^{1}\left(\mathbb{R}^{n}\right)$ was first introduced by Fefferman and Soria in [15]. Then, Liu [26] studied the weak $W H^{p}\left(\mathbb{R}^{n}\right)$ space for $p \in(0, \infty)$ and 
established a weak atomic decomposition for $p \in(0,1]$. Liu in [26] also showed that the $\delta$-Calderón-Zygmund operator is bounded from $H^{p}\left(\mathbb{R}^{n}\right)$ to $W H^{p}\left(\mathbb{R}^{n}\right)$ with $p=n /(n+\delta)$, which was extended to the weighted weak Hardy spaces in [29].

Let $L_{1}$ be a nonnegative self-adjoint operator in $L^{2}\left(\mathbb{R}^{n}\right)$ satisfying the Davies-Gaffney estimates and $L_{2}$ a second order divergence form elliptic operator with complex bounded measurable coefficients. A typical example of $L_{1}$ is the Schrödinger operator $-\Delta+V$, where $0 \leq V \in L_{\text {loc }}^{1}\left(\mathbb{R}^{n}\right)$. Let $H_{L_{i}}^{p}\left(\mathbb{R}^{n}\right)$ be the Hardy space associated to $L_{i}$ for $i \in\{1,2\}$. In this paper, we prove that the Riesz transform $D\left(L_{i}^{-1 / 2}\right)$ is bounded from $H_{L_{i}}^{p}\left(\mathbb{R}^{n}\right)$ to the weak Hardy space $W H^{p}\left(\mathbb{R}^{n}\right)$ in the critical case that $p=n /(n+1)$. To be precise, we have the following general result.

Theorem 1.1. Let $p \equiv n /(n+1), L_{1}$ be a nonnegative self-adjoint operator in $L^{2}\left(\mathbb{R}^{n}\right)$ satisfying the assumptions $\left(A_{1}\right)$ and $\left(A_{2}\right)$ as in Section 2 and $D$ the operator satisfying the assumptions $\left(B_{1}\right),\left(B_{2}\right)$ and $\left(B_{3}\right)$ as in Section 2. Then the operator $D\left(L_{1}^{-1 / 2}\right)$ is bounded from $H_{L_{1}}^{p}\left(\mathbb{R}^{n}\right)$ to the classical weak Hardy space $W H^{p}\left(\mathbb{R}^{n}\right)$. Moreover, there exists a positive constant $C$ such that for all $f \in H_{L_{1}}^{p}\left(\mathbb{R}^{n}\right)$,

$$
\left\|D\left(L_{1}^{-1 / 2}\right) f\right\|_{W H^{p}\left(\mathbb{R}^{n}\right)} \leq C\|f\|_{H_{L_{1}}^{p}\left(\mathbb{R}^{n}\right)} .
$$

As an application of Theorem 1.1, we obtain the boundedness of $\nabla(-\Delta+V)^{-1 / 2}$ with $0 \leq V \in L_{\text {loc }}^{1}\left(\mathbb{R}^{n}\right)$ from $H_{-\Delta+V}^{p}\left(\mathbb{R}^{n}\right)$ to the classical weak Hardy space $W H^{p}\left(\mathbb{R}^{n}\right)$ in the critical case that $p=n /(n+1)$ as follows.

Corollary 1.1. Let $p \equiv n /(n+1)$ and $0 \leq V \in L_{\text {loc }}^{1}\left(\mathbb{R}^{n}\right)$. Then the Riesz transform $\nabla(-\Delta+V)^{-1 / 2}$ is bounded from $H_{-\Delta+V}^{p}\left(\mathbb{R}^{n}\right)$ to $W H^{p}\left(\mathbb{R}^{n}\right)$. Moreover, there exists a positive constant $C$ such that for all $f \in H_{-\Delta+V}^{p}\left(\mathbb{R}^{n}\right)$,

$$
\left\|\nabla(-\Delta+V)^{-1 / 2} f\right\|_{W H^{p}\left(\mathbb{R}^{n}\right)} \leq C\|f\|_{H_{-\Delta+V}^{p}\left(\mathbb{R}^{n}\right)} .
$$

On the Riesz transform defined by the second order divergence form elliptic operator with complex bounded measurable coefficients, we also have the following endpoint boundedness in the critical case that $p \equiv n /(n+1)$.

Theorem 1.2. Let $p \equiv n /(n+1)$ and $L_{2}$ be the second order divergence form elliptic operator with complex bounded measurable coefficients. Then the Riesz transform $\nabla\left(L_{2}^{-1 / 2}\right)$ is bounded from $H_{L_{2}}^{p}\left(\mathbb{R}^{n}\right)$ to $W H^{p}\left(\mathbb{R}^{n}\right)$. Moreover, there exists a positive constant $C$ such that for all $f \in H_{L_{2}}^{p}\left(\mathbb{R}^{n}\right)$,

$$
\left\|\nabla\left(L_{2}^{-1 / 2}\right) f\right\|_{W H^{p}\left(\mathbb{R}^{n}\right)} \leq C\|f\|_{H_{L_{2}}^{p}\left(\mathbb{R}^{n}\right)} .
$$

Recall that the second order divergence form elliptic operator with complex bounded measurable coefficients may not be nonnegative self-adjoint operator in $L^{2}\left(\mathbb{R}^{n}\right)$. Thus, we cannot deduce the conclusion of Theorem 1.2 from Theorem 1.1. However, if $L$ is a second order divergence form elliptic operator with real symmetric bounded measurable coefficients, then $L$ satisfies the assumptions of both Theorem 1.1 and Theorem 1.2. 
We prove Theorems 1.1 and 1.2 by using the characterization of $W H^{p}\left(\mathbb{R}^{n}\right)$ in terms of the radial maximal function, namely, we need estimate the weak $L^{p}\left(\mathbb{R}^{n}\right)$ quasi-norm of the radial maximal function of the Riesz transform acting on the atoms or molecules of the Hardy spaces $H_{L_{i}}^{p}\left(\mathbb{R}^{n}\right)$. Unlike the proof of the endpoint boundedness of the classical Riesz transform $\nabla(-\Delta)^{-1 / 2}$, whose kernel has the pointwise size estimate and regularity, the strategy to show Theorems 1.1 and 1.2 is to divide the radial maximal function into two parts by the time $t$ based on the radius of the associated balls of atoms or molecules and then estimate each part via using $L^{2}$ off-diagonal estimates (see [17, 20] or Lemma 2.1 below).

This paper is organized as follows. In Section 2, we describe some assumptions on the operator $L_{1}$; then we recall some notion and properties concerning the Hardy space associated to $L_{1}$ and second order divergence form elliptic operator $L_{2}$ with complex bounded measurable coefficients. We also recall the definition of weak Hardy spaces and present some technical lemmas which are used later in the next section. Section 3 is devoted to the proof Theorem 1.1, Corollary 1.1, and Theorem 1.2. In Section 4, a similar result on the Riesz transforms defined by higher order divergence form homogeneous elliptic operators with complex bounded measurable coefficients or Schrödinger-type operators is also presented.

Finally, we make some conventions on the notation. Throughout the whole paper, we always let $\mathbb{N} \equiv\{1,2, \cdots\}$ and $\mathbb{Z}_{+} \equiv \mathbb{N} \cup\{0\}$. We use $C$ to denote a positive constant, that is independent of the main parameters involved but whose value may differ from line to line. Constants with subscripts, such as $C_{0}$, do not change in different occurrences. If $f \leq C g$, we then write $f \lesssim g$; and if $f \lesssim g \lesssim f$, we then write $f \sim g$. For all $x \in \mathbb{R}^{n}$ and $r \in(0, \infty)$, let $B(x, r) \equiv\left\{y \in \mathbb{R}^{n}:|x-y|<r\right\}$ and $\alpha B(x, r) \equiv B(x, \alpha r)$ for any $\alpha>0$. Also, for any set $E \in \mathbb{R}^{n}$, we use $E^{\complement}$ to denote the set $\mathbb{R}^{n} \backslash E$ and $\chi_{E}$ the characteristic function of $E$.

\section{Preliminaries}

We begin with recalling some known results on the Hardy spaces associated to operators and the weak Hardy spaces.

Let $L_{1}$ be a linear operator initially defined in $L^{2}\left(\mathbb{R}^{n}\right)$ satisfying the following assumptions:

$\left(\mathrm{A}_{1}\right) L_{1}$ is nonnegative self-adjoint;

$\left(\mathrm{A}_{2}\right)$ The semigroup $\left\{e^{-t L_{1}}\right\}_{t>0}$ generated by $L_{1}$ is analytic on $L^{2}\left(\mathbb{R}^{n}\right)$ and satisfying the Davies-Gaffney estimates, namely, there exist positive constants $C_{1}$ and $C_{2}$ such that for all closed sets $E, F \subset \mathbb{R}^{n}, t \in(0, \infty)$ and $f \in L^{2}\left(\mathbb{R}^{n}\right)$ supported in $E$,

$$
\left\|e^{-t L_{1}} f\right\|_{L^{2}(F)} \leq C_{1} \exp \left\{-\frac{[\operatorname{dist}(E, F)]^{2}}{C_{2} t}\right\}\|f\|_{L^{2}(E)},
$$

where and in what follows, $\operatorname{dist}(E, F) \equiv \inf _{x \in E, y \in F}|x-y|$ is the distance between $E$ and $F$.

Typical examples of operators satisfying assumptions $\left(\mathrm{A}_{1}\right)$ and $\left(\mathrm{A}_{2}\right)$ include the second 
order divergence form elliptic operator with real symmetric bounded measurable coefficients and the Schrödinger operator $-\Delta+V$ with $0 \leq V \in L_{\text {loc }}^{1}\left(\mathbb{R}^{n}\right)$.

Let $\Gamma(x) \equiv\left\{(y, t) \in \mathbb{R}^{n} \times(0, \infty):|x-y|<t\right\}$ be the cone with the vertex $x \in \mathbb{R}^{n}$. For all $f \in L^{2}\left(\mathbb{R}^{n}\right)$ and $x \in \mathbb{R}^{n}$, the $L_{1}$-adapted square function $S_{L_{1}} f(x)$ is defined by

$$
S_{L_{1}} f(x) \equiv\left\{\iint_{\Gamma(x)}\left|t^{2} L_{1} e^{-t^{2} L_{1}} f(y)\right|^{2} \frac{d y d t}{t^{n+1}}\right\}^{1 / 2} .
$$

As in $[16,21]$, we define the Hardy space $H_{L_{1}}^{p}\left(\mathbb{R}^{n}\right)$ associated to the operator $L_{1}$ as follows.

Definition $2.1([16,21])$. Let $p \in(0,1]$ and $L_{1}$ be an operator defined in $L^{2}\left(\mathbb{R}^{n}\right)$ satisfying the assumptions $\left(\mathrm{A}_{1}\right)$ and $\left(\mathrm{A}_{2}\right)$. A function $f \in L^{2}\left(\mathbb{R}^{n}\right)$ is said to be in $\mathbb{H}_{L_{1}}^{p}\left(\mathbb{R}^{n}\right)$ if $S_{L_{1}} f \in L_{1}^{p}\left(\mathbb{R}^{n}\right)$; moreover, define $\|f\|_{H_{L_{1}}^{p}\left(\mathbb{R}^{n}\right)} \equiv\left\|S_{L_{1}} f\right\|_{L^{p}\left(\mathbb{R}^{n}\right)}$. The Hardy space $H_{L_{1}}^{p}\left(\mathbb{R}^{n}\right)$ is then defined to be the completion of $\mathbb{H}_{L_{1}}^{p}\left(\mathbb{R}^{n}\right)$ with respect to the quasi-norm $\|\cdot\|_{H_{L_{1}}^{p}\left(\mathbb{R}^{n}\right)}$.

For all $p \in(0,1]$ and $M \in \mathbb{N}$, a function $a \in L^{2}\left(\mathbb{R}^{n}\right)$ is called a $(p, 2, M)_{L_{1}}$-atom if there exists a function $b \in D\left(L_{1}^{M}\right)$ and a ball $B \equiv B\left(x_{B}, r_{B}\right) \subset \mathbb{R}^{n}$ such that

(i) $a=L_{1}^{M} b$;

(ii) for each $\ell \in\{0,1, \cdots, M\}, \operatorname{supp} L_{1}^{\ell} b \subset B$;

(iii) for all $\ell \in\{0,1, \cdots, M\}$,

$$
\left\|\left(r_{B}^{2} L_{1}\right)^{k} b\right\|_{L^{2}\left(\mathbb{R}^{n}\right)} \leq r_{B}^{2 M+n\left(\frac{1}{2}-\frac{1}{p}\right)} .
$$

We then have the following atomic decomposition of $H_{L_{1}}^{p}\left(\mathbb{R}^{n}\right)$.

Theorem 2.1 ([16, 21]). Let $p \in(0,1]$. Suppose that $M \in \mathbb{N}$ and $M>\frac{n}{2}\left(\frac{1}{p}-\frac{1}{2}\right)$. Then for all $f \in L^{2}\left(\mathbb{R}^{n}\right) \cap H_{L_{1}}^{p}\left(\mathbb{R}^{n}\right)$, there exist a sequence $\left\{a_{j}\right\}_{j=0}^{\infty}$ of $(p, 2, M)_{L_{1}}$-atoms and a sequence $\left\{\lambda_{j}\right\}_{j=0}^{\infty}$ of numbers such that $f=\sum_{j=0}^{\infty} \lambda_{j} a_{j}$ in both $H_{L_{1}}^{p}\left(\mathbb{R}^{n}\right)$ and $L^{2}\left(\mathbb{R}^{n}\right)$, and $\|f\|_{H_{L_{1}}^{p}\left(\mathbb{R}^{n}\right)} \sim\left\{\sum_{j=0}^{\infty}\left|\lambda_{j}\right|^{p}\right\}^{1 / p}$.

For the second order divergence form operator, the associated Hardy space were studied in $[18,19,20,23]$. More precisely, let $L_{2} \equiv-\operatorname{div}(\mathrm{A} \nabla)$ be a second order divergence form elliptic operator with complex bounded measurable coefficients. We say that $L_{2}$ is elliptic if the matrix $A \equiv\left\{a_{i, j}\right\}_{i, j=1}^{n}$ satisfying the elliptic condition, namely, there exist positive constants $0<\lambda \leq \Lambda<\infty$ such that $\lambda|\xi|^{2} \leq \Re(A \xi \cdot \bar{\xi})$ and $|A \xi \cdot \bar{\xi}| \leq \Lambda|\xi|^{2}$, where for any $z \in \mathbb{C}, \Re z$ denotes the real part of $z$.

Definition $2.2([18,20,23])$. Let $p \in(0,1]$ and $L_{2}$ be the second order divergence form elliptic operator with complex bounded measurable coefficients. A function $f \in L^{2}\left(\mathbb{R}^{n}\right)$ is said to be in $\mathbb{H}_{L_{2}}^{p}\left(\mathbb{R}^{n}\right)$ if $S_{L_{2}} f \in L^{p}\left(\mathbb{R}^{n}\right)$; moreover, define $\|f\|_{H_{L_{2}}^{p}\left(\mathbb{R}^{n}\right)} \equiv\left\|S_{L_{2}} f\right\|_{L^{p}\left(\mathbb{R}^{n}\right)}$. The Hardy space $H_{L_{2}}^{p}\left(\mathbb{R}^{n}\right)$ is then defined to be the completion of $\mathbb{H}_{L_{2}}^{p}\left(\mathbb{R}^{n}\right)$ with respect to the quasi-norm $\|\cdot\|_{H_{L_{2}}^{p}\left(\mathbb{R}^{n}\right)}$. 
Recall that in $[20,23]$, for all $p \in(0,1], \epsilon \in(0, \infty)$ and $M \in \mathbb{N}$, a function $A \in L^{2}\left(\mathbb{R}^{n}\right)$ is called an $\left(H_{L_{2}}^{p}, \epsilon, M\right)$-molecule if there exists a ball $B \equiv B\left(x_{B}, r_{B}\right) \subset \mathbb{R}^{n}$ such that

(i) for each $\ell \in\{1, \cdots, M\}, A$ belongs to the range of $L_{2}^{\ell}$ in $L^{2}\left(\mathbb{R}^{n}\right)$;

(ii) for all $i \in \mathbb{Z}_{+}$and $\ell \in\{0,1, \cdots, M\}$,

$$
\left\|\left(r_{B}^{2} L_{2}\right)^{-\ell} A\right\|_{L^{2}\left(S_{i}(B)\right)} \leq\left(2^{i} r_{B}\right)^{n\left(\frac{1}{2}-\frac{1}{p}\right)} 2^{-i \epsilon},
$$

where $S_{0}(B) \equiv B$ and $S_{i}(B) \equiv 2^{i} B \backslash 2^{i-1} B$ for all $i \in \mathbb{N}$.

Assume that $\left\{m_{j}\right\}_{j}$ is a sequence of $\left(H_{L_{2}}^{p}, \epsilon, M\right)$-molecules and $\left\{\lambda_{j}\right\}_{j}$ a sequence of numbers satisfying $\sum_{j}\left|\lambda_{j}\right|^{p}<\infty$. For any $f \in L^{2}\left(\mathbb{R}^{n}\right)$, if $f=\sum_{j} \lambda_{j} m_{j}$ in $L^{2}\left(\mathbb{R}^{n}\right)$, then $\sum_{j} \lambda_{j} m_{j}$ is called a molecular $\left(H_{L_{2}}^{p}, 2, \epsilon, M\right)$-representation of $f$. The molecular Hardy space $H_{L_{2}, \mathrm{~mol}, M}^{p}\left(\mathbb{R}^{n}\right)$ is then defined to be the completion of the space

$$
\mathbb{H}_{L_{2}, \mathrm{~mol}, M}^{p}\left(\mathbb{R}^{n}\right) \equiv\left\{f: f \text { has a molecular }\left(H_{L_{2}}^{p}, 2, \epsilon, M\right) \text {-representation }\right\}
$$

with respect to the quasi-norm

$$
\begin{array}{r}
\|f\|_{H_{L_{2}, \mathrm{~mol}, M}^{p}\left(\mathbb{R}^{n}\right)} \equiv \inf \left\{\left(\sum_{j=0}^{\infty}\left|\lambda_{j}\right|^{p}\right)^{1 / p}: f=\sum_{j=0}^{\infty} \lambda_{j} A_{j}\right. \text { is a molecular } \\
\left.\left(H_{L_{2}}^{p}, 2, \epsilon, M\right) \text {-representation }\right\}
\end{array}
$$

where the infimum is taken over all the molecular $\left(H_{L_{2}}^{p}, 2, \epsilon, M\right)$-representations of $f$ as above.

We have the following molecular characterization of $H_{L_{2}}^{p}\left(\mathbb{R}^{n}\right)$.

Theorem $2.2([20,23])$. Let $p \in(0,1]$. Suppose that $M>\frac{n}{2}\left(\frac{1}{p}-\frac{1}{2}\right)$ and $\epsilon>0$. Then $H_{L_{2}}^{p}\left(\mathbb{R}^{n}\right)=H_{L_{2}, \mathrm{~mol}, M}^{p}\left(\mathbb{R}^{n}\right)$. Moreover, $\|f\|_{H_{L_{2}}^{p}\left(\mathbb{R}^{n}\right)} \sim\|f\|_{H_{L_{2}, \mathrm{~mol}, M}^{p}\left(\mathbb{R}^{n}\right)}$, where the implicit constants depend only on $M, n, p, \epsilon$ and the constants appearing in the ellipticity.

We now recall the definition of the weak Hardy space (see, for example, [15, 26, 27]). Let $p \in(0,1]$ and $\varphi \in \mathcal{S}\left(\mathbb{R}^{n}\right)$ with support in the unit ball $B(0,1)$. The weak Hardy space $W H^{p}\left(\mathbb{R}^{n}\right)$ is defined to be the space

$$
\left\{f \in \mathcal{S}^{\prime}\left(\mathbb{R}^{n}\right):\|f\|_{W H^{p}\left(\mathbb{R}^{n}\right)} \equiv \sup _{\alpha>0}\left(\alpha^{p}\left|\left\{x \in \mathbb{R}^{n}: \sup _{t>0}\left|\varphi_{t} * f(x)\right|>\alpha\right\}\right|\right)^{1 / p}<\infty\right\} .
$$

Let $L_{1}$ be a nonnegative self-adjoint operator in $L^{2}\left(\mathbb{R}^{n}\right)$ satisfying the assumptions $\left(\mathrm{A}_{1}\right)$ and $\left(\mathrm{A}_{2}\right)$. Following [1], let the operator $D$ be a linear operator defined densely in $L^{2}\left(\mathbb{R}^{n}\right)$ and satisfy the following assumptions:

( $\left.\mathrm{B}_{1}\right) D L_{1}^{-1 / 2}$ is bounded on $L^{2}\left(\mathbb{R}^{n}\right)$;

$\left(\mathrm{B}_{2}\right)$ the family of operators, $\left\{\sqrt{t} D e^{-t L_{1}}\right\}_{t>0}$, satisfy the Davies-Gaffney estimates as in $(2.1)$; 
$\left(\mathrm{B}_{3}\right)$ for all $(p, 2, M)_{L_{1}}$-atoms $a, \int_{\mathbb{R}^{n}} D L_{1}^{-1 / 2} a(x) d x=0$.

Typical examples of $D$ and $L_{1}$ satisfying the assumptions $\left(\mathrm{B}_{1}\right),\left(\mathrm{B}_{2}\right)$ and $\left(\mathrm{B}_{3}\right)$ include that $D$ is the gradient operator $\nabla$ on $\mathbb{R}^{n}$, and $L_{1}$ is the second order divergence form elliptic operator with real symmetric bounded measurable coefficients or the Schrödinger operator $-\Delta+V$ with $0 \leq V \in L_{\text {loc }}^{1}\left(\mathbb{R}^{n}\right)$ as proved below.

Lemma 2.1. Let $0 \leq V \in L_{\text {loc }}^{1}\left(\mathbb{R}^{n}\right)$. Then the Schrödinger operator $T \equiv-\Delta+V$ satisfies the assumptions $\left(A_{1}\right)$ and $\left(A_{2}\right)$, and both $T$ and the gradient operator $\nabla$ satisfy the assumptions $\left(B_{1}\right),\left(B_{2}\right)$ and $\left(B_{3}\right)$.

Proof. It is easy to see that $T$ is nonnegative self-adjoint.

Let $e^{-t T}(\cdot, \cdot)$ be the integral kernel of the semigroup $e^{-t T}$. By Trotter's formula (see, for example, [34]), we know that for all $t \in(0, \infty)$ and $x, y \in \mathbb{R}^{n}$,

$$
0 \leq e^{-t T}(x, y) \leq e^{-t \Delta}(x, y) \sim t^{-\frac{n}{2}} \exp \left\{-\frac{|x-y|^{2}}{t}\right\}
$$

which implies that the semigroup $\left\{e^{-t T}\right\}_{t>0}$ satisfies (2.1). Thus, $T$ satisfies the assumptions $\left(\mathrm{A}_{1}\right)$ and $\left(\mathrm{A}_{2}\right)$.

Moreover, by [16, Lemma 8.5], we conclude that there exists a positive constant $C_{2}$ such that for for all closed sets $E, F \subset \mathbb{R}^{n}, t \in(0, \infty)$ and $f \in L^{2}\left(\mathbb{R}^{n}\right)$ supported in $E$,

$$
\left\|t \nabla e^{-t^{2} T} f\right\|_{L^{2}(F)} \lesssim \exp \left\{-\frac{[\operatorname{dist}(E, F)]^{2}}{C_{2} t^{2}}\right\}\|f\|_{L^{2}(E)},
$$

which, combining the $L^{2}\left(\mathbb{R}^{n}\right)$-boundedness of the Riesz transform $\nabla\left(T^{-1 / 2}\right)$ (see $[16$, (8.20)]) and the fact that $\int_{\mathbb{R}^{n}} \nabla\left(T^{-1 / 2}\right) a(y) d y=0$ (see, for example [16, 21]), implies that both $T$ and the gradient operator satisfy the assumptions $\left(B_{1}\right),\left(B_{2}\right)$ and $\left(B_{3}\right)$. This finishes the proof of Lemma 2.1.

We also need the following technical lemmas.

Lemma $2.2([27,31])$. Let $p \in(0,1)$ and $\left\{f_{j}\right\}_{j}$ be a sequence of measurable functions. If $\sum_{j}\left|\lambda_{j}\right|^{p}<\infty$ and there exists a positive constant $\widetilde{C}$ such that for all $\left\{f_{j}\right\}_{j}$ and $\alpha \in(0, \infty)$, $\left|\left\{x \in \mathbb{R}^{n}:\left|f_{j}\right|>\alpha\right\}\right| \leq \widetilde{C} \alpha^{-p}$. Then, for all $\alpha \in(0, \infty)$,

$$
\left|\left\{x \in \mathbb{R}^{n}:\left|\sum_{j} \lambda_{j} f_{j}(x)\right|>\alpha\right\}\right| \leq \widetilde{C} \frac{2-p}{1-p} \alpha^{-p} \sum_{j}\left|\lambda_{j}\right|^{p} .
$$

Lemma $2.3([1,17])$. Let $L_{1}$ be a nonnegative self-adjoint operator satisfying the assumptions $\left(A_{1}\right)$ and $\left(A_{2}\right)$ and $D$ the operator satisfying the assumptions $\left(B_{1}\right),\left(B_{2}\right)$ and $\left(B_{3}\right)$. Let $M \in \mathbb{N}$. Then there exists a positive constant $C$, depending on $M$, such that for all closed sets $E, F$ in $\mathbb{R}^{n}$ with $\operatorname{dist}(E, F)>0, f \in L^{2}\left(\mathbb{R}^{n}\right)$ supported in $E$ and $t \in(0, \infty)$,

$$
\left\|D L_{1}^{-1 / 2}\left(I-e^{-t L_{1}}\right)^{M} f\right\|_{L^{2}(F)} \leq C\left(\frac{t}{[\operatorname{dist}(E, F)]^{2}}\right)^{M}\|f\|_{L^{2}(E)}
$$


and

$$
\left\|D L_{1}^{-1 / 2}\left(t L_{1} e^{-t L_{1}}\right)^{M} f\right\|_{L^{2}(F)} \leq C\left(\frac{t}{[\operatorname{dist}(E, F)]^{2}}\right)^{M}\|f\|_{L^{2}(E)} .
$$

Moreover, if $L_{2}$ is a second order divergence form elliptic operator with complex bounded measurable coefficients, then (2.4) and (2.5) still hold when $D$ and $L_{1}$ are replaced, respectively, by the gradient operator $\nabla$ and $L_{2}$.

\section{Proofs of main results}

In this section, we show Theorem 1.1, Corollary 1.1 and Theorem 1.2.

Proof of Theorem 1.1. Let $p \equiv \frac{n}{n+1}$. By the density of $H_{L_{1}}^{p}\left(\mathbb{R}^{n}\right) \cap L^{2}\left(\mathbb{R}^{n}\right)$ in $H_{L_{1}}^{p}\left(\mathbb{R}^{n}\right)$, we only need consider $f \in H_{L_{1}}^{p}\left(\mathbb{R}^{n}\right) \cap L^{2}\left(\mathbb{R}^{n}\right)$. Let $M \in \mathbb{N}$ and $M>\max \left\{\frac{1}{2}+\frac{n}{4}, 1\right\}$. By Theorem 2.1, we know that there exist a sequence $\left\{a_{j}\right\}_{j}$ of $(p, 2, M)_{L_{1}}$-atoms and a sequence $\left\{\lambda_{j}\right\}_{j}$ of numbers such that

$$
f=\sum_{j} \lambda_{j} a_{j}
$$

in $L^{2}\left(\mathbb{R}^{n}\right)$ and $\|f\|_{H_{L_{1}}^{p}\left(\mathbb{R}^{n}\right)} \sim\left\{\sum_{j}\left|\lambda_{j}\right|^{p}\right\}^{1 / p}$. To show Theorem 1.1, by (3.1) and the definition of $W H^{p}\left(\mathbb{R}^{n}\right)$, we see that it suffices to prove that for all $\alpha \in(0, \infty)$,

$$
\left|\left\{x \in \mathbb{R}^{n}: \sup _{0<t<\infty}\left|\varphi_{t} *\left(\sum_{j} \lambda_{j} D L_{1}^{-1 / 2} a_{j}\right)(x)\right|>\alpha\right\}\right| \lesssim \frac{1}{\alpha^{p}} \sum_{j}\left|\lambda_{j}\right|^{p},
$$

where $\varphi \in C_{c}^{\infty}\left(\mathbb{R}^{n}\right)$ satisfies $\operatorname{supp} \varphi \subset B(0,1)$, and for all $x \in \mathbb{R}^{n}$ and $t \in(0, \infty), \varphi_{t}(x) \equiv$ $\frac{1}{t^{n}} \varphi\left(\frac{x}{t}\right)$. In order to prove (3.2), by Lemma 2.2 , it suffices to show that for any $(p, 2, M)_{L_{1}}$ atom $a$ associated with the ball $B \equiv B\left(x_{B}, r_{B}\right)$ and $\alpha \in(0, \infty)$,

$$
\left|\left\{x \in \mathbb{R}^{n}: \sup _{0<t<\infty}\left|\varphi_{t} *\left(D L_{1}^{-1 / 2} a\right)(x)\right|>\alpha\right\}\right| \lesssim \frac{1}{\alpha^{p}}
$$

Let $\mathcal{M}$ be the Hardy-Littlewood maximal function. It is easy to see that

$$
\sup _{0<t<\infty}\left|\varphi_{t} *\left(D L_{1}^{-1 / 2} a\right)\right| \lesssim \mathcal{M}\left(D L_{1}^{-1 / 2} a\right)
$$

Then by Chebyshev's inequality, Hölder's inequality, the $L^{2}\left(\mathbb{R}^{n}\right)$-boundedness of $\mathcal{M}$, the $L^{2}\left(\mathbb{R}^{n}\right)$-boundedness of $D L_{1}^{-1 / 2}$ via $\left(\mathrm{B}_{1}\right)$, and $(2.2)$, we know that

$$
\begin{aligned}
& \left|\left\{x \in 16 B: \sup _{0<t<\infty}\left|\varphi_{t} *\left(D L_{1}^{-1 / 2} a\right)(x)\right|>\alpha\right\}\right| \\
& \quad \lesssim \frac{1}{\alpha^{p}}\left\|\sup _{0<t<\infty}\left|\varphi_{t} *\left(D L_{1}^{-1 / 2} a\right)\right|\right\|_{L^{p}(16 B)}^{p} \lesssim \frac{1}{\alpha^{p}}\left\|\mathcal{M}\left(D L_{1}^{-1 / 2} a\right)\right\|_{L^{p}(16 B)}^{p}
\end{aligned}
$$


Jun Cao, Dachun Yang and Sibei Yang

$$
\lesssim \frac{1}{\alpha^{p}}\left\|\mathcal{M}\left(D L_{1}^{-1 / 2} a\right)\right\|_{L^{2}\left(\mathbb{R}^{n}\right)}^{p}|B|^{1-\frac{p}{2}} \lesssim \frac{1}{\alpha^{p}}\|a\|_{L^{2}\left(\mathbb{R}^{n}\right)}^{p}|B|^{1-\frac{p}{2}} \lesssim \frac{1}{\alpha^{p}}
$$

On the other hand, we have

$$
\begin{aligned}
& \left\{x \in(16 B)^{\complement}: \sup _{0<t<\infty}\left|\varphi_{t} *\left(D L_{1}^{-1 / 2} a\right)(x)\right|>\alpha\right\} \\
& \subset\left\{x \in(16 B)^{\complement}: \sup _{0<t<r_{B}}\left|\varphi_{t} *\left(D L_{1}^{-1 / 2} a\right)(x)\right|>\alpha / 2\right\} \\
& \bigcup\left\{x \in(16 B)^{\complement}: \sup _{r_{B}<t<\infty}|\cdots|>\alpha / 2\right\} \equiv \mathrm{I} \cup \mathrm{J} .
\end{aligned}
$$

To estimate I, let $S_{i}(B) \equiv 2^{i} B \backslash 2^{i-1} B$ and $\widetilde{S}_{i}(B) \equiv 2^{i+1} B \backslash 2^{i-2} B$ with $i \in \mathbb{N}$. For all $i \geq 5, x \in S_{i}(B)$ and $y \in B\left(x, r_{B}\right)$, from $\operatorname{supp} \varphi \subset B(0,1)$, it follows that $y \in \widetilde{S}_{i}(B)$. For $i \geq 5$, let

$$
\mathrm{I}_{i} \equiv\left\{x \in S_{i}(B): \sup _{0<t<r_{B}}\left|\varphi_{t} *\left(D L_{1}^{-1 / 2} a\right)(x)\right|>\alpha / 2\right\}
$$

By Chebyshev's inequality, Hölder's inequality, the $L^{2}\left(\mathbb{R}^{n}\right)$-boundedness of $\mathcal{M}$, Lemma 2.3 and (2.2), we conclude that

$$
\begin{aligned}
& \left|\mathrm{I}_{i}\right| \lesssim \alpha^{-p} \int_{S_{i}(B)}\left[\sup _{0<t<r_{B}}\left|\int_{\widetilde{S}_{i}(B)} t^{-n} \varphi\left(\frac{x-y}{t}\right)\left[\chi_{\widetilde{S}_{i}(B)}(y) D L_{1}^{-1 / 2} a(y)\right] d y\right|\right]^{p} d x \\
& \lesssim \alpha^{-p} \int_{S_{i}(B)}\left[\mathcal{M}\left(\chi_{\widetilde{S}_{i}(B)} D L_{1}^{-1 / 2} a\right)(x)\right]^{p} d x \\
& \lesssim \alpha^{-p}\left|S_{i}(B)\right|^{1-p / 2}\left\|D L_{1}^{-1 / 2} a\right\|_{L^{2}\left(\widetilde{S}_{i}(B)\right)}^{p} \\
& \lesssim \alpha^{-p}\left|S_{i}(B)\right|^{1-p / 2}\left[\left\|D L_{1}^{-1 / 2}\left(I-e^{-r_{B}^{2} L_{1}}\right)^{M} a\right\|_{L^{2}\left(\widetilde{S}_{i}(B)\right)}^{p}\right. \\
& \left.+\sum_{k=1}^{M}\left\|D L_{1}^{-1 / 2}\left(r_{B}^{2} L_{1} e^{-\frac{k}{M} r_{B}^{2} L_{1}}\right)^{M} r_{B}^{-2 M} b\right\|_{L^{2}\left(\widetilde{S}_{i}(B)\right)}^{p}\right] \\
& \lesssim \alpha^{-p}\left|S_{i}(B)\right|^{1-p / 2}\left[\frac{r_{B}^{2}}{\left(2^{i} r_{B}\right)^{2}}\right]^{M p}|B|^{p / 2-1} \sim 2^{-i[2 M p-n(1-p / 2)]} \alpha^{-p} .
\end{aligned}
$$

From this, the definition of $\mathrm{I}_{i}, p=\frac{n}{n+1}$ and $M>\frac{1}{2}+\frac{n}{4}$, we deduce that $|\mathrm{I}| \lesssim \sum_{i=1}^{\infty}\left|\mathrm{I}_{i}\right| \lesssim \frac{1}{\alpha^{p}}$, which is a desired estimate for I.

To estimate $\mathrm{J}$, by the assumption that $\int_{\mathbb{R}^{n}} D L_{1}^{-\frac{1}{2}} a(y) d y=0$ via $\left(\mathrm{B}_{3}\right)$, we know that

$$
\begin{aligned}
|\mathrm{J}| \lesssim \mid & \left\{x \in(16 B)^{\complement}:\right. \\
& \left.\sum_{i=0}^{\infty} \sup _{r_{B}<t<\infty}\left|\int_{S_{i}(B)} \frac{1}{t^{n}}\left[\varphi\left(\frac{x-y}{t}\right)-\varphi\left(\frac{x-x_{B}}{t}\right)\right] D L_{1}^{-\frac{1}{2}} a(y) d y\right|>\alpha / 2\right\} \mid .
\end{aligned}
$$


Let $F_{i}(x) \equiv \sup _{r_{B}<t<\infty}\left|\int_{S_{i}(B)} \frac{1}{t^{n}}\left[\varphi\left(\frac{x-y}{t}\right)-\varphi\left(\frac{x-x_{B}}{t}\right)\right] D L_{1}^{-\frac{1}{2}} a(y) d y\right|$ and

$$
\mathrm{J}_{i} \equiv\left\{x \in(16 B)^{\complement}: F_{i}(x)>\alpha / 2\right\}
$$

To obtain a desired estimate for J, by Lemma 2.2, it suffices to show that there exists a positive constant $C_{0}$ such that

$$
\left|\mathrm{J}_{i}\right| \lesssim \frac{2^{-C_{0} i}}{\alpha^{p}}
$$

From the mean value theorem, Hölder's inequality, $\operatorname{supp} \varphi \subset B(0,1)$, Lemma 2.3 and (2.2), we infer that

$$
\begin{aligned}
F_{i}(x) \leq & \sup _{j \in \mathbb{Z}_{+} 2^{j} r_{B} \leq t<2^{j+1} r_{B}} \chi_{\left(2^{i+1}+2^{j+1}\right) B}(x) \int_{S_{i}(B)} \frac{1}{t^{n}}\|\nabla \varphi\|_{L^{\infty}\left(\mathbb{R}^{n}\right)}\left|\frac{y-x_{B}}{t}\right|\left|D L_{1}^{-\frac{1}{2}} a(y)\right| d y \\
\lesssim & \sup _{j \in \mathbb{Z}_{+}} \chi_{\left(2^{i+1}+2^{j+1}\right) B}(x) \sup _{2^{j} r_{B} \leq t<2^{j+1} r_{B}} 2^{-j(n+1)}|B|^{-1} 2^{i}\left|S_{i}(B)\right|^{1 / 2} \\
& \times\left\|D L_{1}^{-\frac{1}{2}} a\right\|_{L^{2}\left(S_{i}(B)\right)} \\
\lesssim & \sup _{j \in \mathbb{Z}_{+}} \chi_{\left(2^{i+1}+2^{j+1}\right) B}(x) \sup _{2^{j} r_{B} \leq t<2^{j+1} r_{B}} 2^{-j(n+1)} 2^{i(n / 2+1)}\left[\frac{r_{B}^{2}}{\left(2^{i} r_{B}\right)^{2}}\right]^{M}|B|^{-1 / p} \\
\equiv & C_{3} \sup _{j \in \mathbb{Z}_{+}} \chi_{\left(2^{i+1}+2^{j+1}\right) B}(x) \sup _{2^{j} r_{B} \leq t<2^{j+1} r_{B}} 2^{-j(n+1)} 2^{-i(2 M-n / 2-1)}|B|^{-1 / p} .
\end{aligned}
$$

Let

$$
j_{0} \equiv \max \left\{j \in \mathbb{Z}_{+}: C_{3} 2^{-j(n+1)} 2^{-i(2 M-n / 2-1)}|B|^{-1 / p}>\alpha / 2\right\} .
$$

For all $x \in\left[\left(2^{i+1}+2^{j_{0}+1}\right) B\right]^{\complement}$, we see that

$$
F_{i}(x) \leq C_{3} \sup _{j \geq j_{0}} \chi_{\left(2^{i+1}+2^{j+1}\right) B}(x) \sup _{2^{j} r_{B} \leq t<2^{j+1} r_{B}} 2^{-j(n+1)} 2^{-i(2 M-n / 2-1)}|B|^{-1 / p} \leq \alpha / 2,
$$

which implies that $x \in \mathrm{J}_{i}^{\complement}$. Thus, $\mathrm{J}_{i} \subset\left(2^{i+1}+2^{j_{0}+1}\right) B$. From this and Chebyshev's inequality, we then deduce that

$$
\left|\mathrm{J}_{i}\right| \lesssim \alpha^{-p} \int_{\left(2^{i+1}+2^{j_{0}+1}\right) B} 2^{-p j_{0}(n+1)} 2^{-i p(2 M-1+n)}|B|^{-1} d x \lesssim 2^{-i[(2 M-1) p-n(1-p)]} \alpha^{-p},
$$

which implies that $(3.3)$ holds with $C_{0} \equiv(2 M-1) p-n(1-p)$. Observe that $C_{0}>0$, since $M>1$ and $p=\frac{n}{n+1}$. Thus, combining the estimate of I and J, we then complete the proof of Theorem 1.1.

Proof of Corollary 1.1. From Lemma 2.1, we deduce that the Schrödinger operator $-\Delta+V$ with $0 \leq V \in L_{\text {loc }}^{1}\left(\mathbb{R}^{n}\right)$ satisfies the assumptions $\left(\mathrm{A}_{1}\right)$ and $\left(\mathrm{A}_{2}\right)$ as in Section 2 , and both $-\Delta+V$ and the gradient operator $\nabla$ satisfy the assumptions $\left(\mathrm{B}_{1}\right),\left(\mathrm{B}_{2}\right)$ and $\left(\mathrm{B}_{3}\right)$ as in Section 2. Thus, from Theorem 1.1, we deduce that the Riesz transform $\nabla(-\Delta+V)^{-1 / 2}$ is bounded from $H_{-\Delta+V}^{p}\left(\mathbb{R}^{n}\right)$ to the classical weak Hardy space $W H^{p}\left(\mathbb{R}^{n}\right)$ in the critical case that $p=n /(n+1)$, which completes the proof of Corollary 1.1. 
Proof of Theorem 1.2. Let $p=\frac{n}{n+1}$ and $M \in \mathbb{N}$ satisfy $M>\frac{n}{4}+\frac{1}{2}$. To prove Theorem 1.2, similar to the proof of Theorem 1.1, by Theorem 2.2 and Lemma 2.2, for each $\left(H_{L}^{p}, \epsilon, M\right)$ molecule $A$ associated to the ball $B\left(x_{B}, r_{B}\right), m \in \mathbb{Z}_{+}$and $\alpha \in(0, \infty)$, we only need estimate the measure of the following sets:

$$
\widetilde{\mathrm{I}} \equiv\left\{x \in(16 B)^{\complement}: \sup _{0<t<r_{B}}\left|\varphi_{t} *\left(\nabla L_{2}^{-1 / 2} A\right)(x)\right|>\alpha / 2\right\}
$$

and

$$
\widetilde{J} \equiv\left\{x \in(16 B)^{\complement}: \sup _{r_{B} \leq t<\infty}\left|\varphi_{t} *\left(\nabla L_{2}^{-1 / 2} A\right)(x)\right|>\alpha / 2\right\}
$$

The estimate of $\widetilde{I}$ is similar to that of $\mathrm{I}$ in the proof of Theorem 1.1. We omit the details. Now we estimate $\widetilde{J}$. Since

$$
\begin{aligned}
|\widetilde{J}| \lesssim & \mid\left\{x \in(16 B)^{\complement}: \sum_{i=0}^{\infty} \sup _{B} \leq t<\infty\right. \\
& \mid \int_{S_{i}(B)} \frac{1}{t^{n}}\left[\varphi\left(\frac{x-y}{t}\right)-\varphi\left(\frac{x-x_{B}}{t}\right)\right] \\
& \left.\times \nabla L_{2}^{-\frac{1}{2}}\left(I-e^{-r_{B}^{2} L_{2}}\right)^{M} A(y) d y \mid>\alpha / 2\right\} \mid \\
& +\mid\left\{x \in(16 B)^{\complement}: \sum_{i=0}^{\infty} \sum_{k=1}^{M} \sup _{r_{B} \leq t<\infty} \mid \int_{S_{i}(B)} \frac{1}{t^{n}}\left[\varphi\left(\frac{x-y}{t}\right)-\varphi\left(\frac{x-x_{B}}{t}\right)\right]\right. \\
& \left.\times \nabla L_{2}^{-\frac{1}{2}}\left(r_{B}^{2} L_{2} e^{-\frac{k}{M} r_{B}^{2} L_{2}}\right)^{M}\left(r_{B}^{2} L_{2}\right)^{-M} A(y) d y \mid>\alpha / 2\right\} .
\end{aligned}
$$

Let $\widetilde{F_{1, i}}(x) \equiv \sup _{r_{B} \leq t<\infty}\left|\int_{S_{i}(B)} \frac{1}{t^{n}}\left[\varphi\left(\frac{x-y}{t}\right)-\varphi\left(\frac{x-x_{B}}{t}\right)\right] \nabla L_{2}^{-\frac{1}{2}}\left(I-e^{-r_{B}^{2} L_{2}}\right)^{M} A(y) d y\right|$,

$$
\begin{aligned}
\widetilde{F_{2, i}}(x) \equiv & \sum_{k=1}^{M} \sup _{r_{B} \leq t<\infty} \mid \int_{S_{i}(B)} \frac{1}{t^{n}}\left[\varphi\left(\frac{x-y}{t}\right)-\varphi\left(\frac{x-x_{B}}{t}\right)\right] \\
& \times \nabla L_{2}^{-\frac{1}{2}}\left(r_{B}^{2} L_{2} e^{-\frac{k}{M} r_{B}^{2} L_{2}}\right)^{M}\left(r_{B}^{2} L_{2}\right)^{-M} A(y) d y \mid,
\end{aligned}
$$

$\widetilde{\mathrm{J}_{1, k}} \equiv\left\{x \in(16 B)^{\complement}: \widetilde{F_{1, i}}(x)>\alpha / 2\right\}$ and $\widetilde{\mathrm{J}_{2, k}} \equiv\left\{x \in(16 B)^{\complement}: \widetilde{F_{2, i}}(x)>\alpha / 2\right\}$. By Lemma 2.2, it suffices to show that there exist positive constants $C_{4}$ and $C_{5}$ such that for all $\alpha \in(0, \infty),\left|\widetilde{\mathrm{J}_{1, k}}\right| \lesssim \frac{2^{-C_{4}}}{\alpha^{p}}$ and $\left|\widetilde{\mathrm{J}_{2, k}}\right| \lesssim \frac{2^{-C_{5} i}}{\alpha^{p}}$. We only prove the first inequality, the proof of the second inequality is similar. Take $\epsilon \in(n+1-1 /(n+1), \infty)$. By the mean value theorem, Hölder's inequality, Lemma 2.3, (2.3) and $\operatorname{supp} \varphi \subset B(0,1)$, we conclude that

$$
\begin{aligned}
\widetilde{F_{1, i}}(x) \lesssim & \sup _{j \in \mathbb{Z}_{+}} \chi_{\left(2^{i+1}+2^{j+1}\right) B}(x) \sup _{2^{j} r_{B} \leq t<2^{j+1} r_{B}} \int_{S_{i}(B)} \frac{1}{t^{n}}\|\nabla \varphi\|_{L^{\infty}\left(\mathbb{R}^{n}\right)}\left|\frac{y-x_{B}}{t}\right| \\
& \times\left|\nabla L_{2}^{-\frac{1}{2}}\left(I-e^{-r_{B}^{2} L_{2}}\right)^{M} A(y)\right| d y
\end{aligned}
$$




$$
\begin{aligned}
\lesssim & \sup _{j \in \mathbb{Z}_{+}} \chi_{\left(2^{i+1}+2^{j+1}\right) B}(x) \sup _{2^{j} r_{B} \leq t<2^{j+1} r_{B}} \int_{S_{i}(B)} \frac{1}{t^{n}}\|\nabla \varphi\|_{L^{\infty}\left(\mathbb{R}^{n}\right)}\left|\frac{y-x_{B}}{t}\right| \\
& \times\left|\nabla L_{2}^{-\frac{1}{2}}\left(I-e^{-r_{B}^{2} L_{2}}\right)^{M}\left(\chi_{\widetilde{S}_{i}(B)} A\right)(y)\right| d y \\
& +\sup _{j \in \mathbb{Z}_{+}} \chi_{\left(2^{i+1}+2^{j+1}\right) B}(x) \sup _{2^{j} r_{B} \leq t<2^{j+1} r_{B}} \int_{S_{i}(B)} \frac{1}{t^{n}}\|\nabla \varphi\|_{L^{\infty}\left(\mathbb{R}^{n}\right)}\left|\frac{y-x_{B}}{t}\right| \\
& \times\left|\nabla L_{2}^{-\frac{1}{2}}\left(I-e^{-r_{B}^{2} L_{2}}\right)^{M}\left(\chi_{\mathbb{R}^{n} \backslash \widetilde{S}_{i}(B)} A\right)(y)\right| d y \\
\lesssim & \sup _{j \in \mathbb{Z}_{+}} \chi_{\left(2^{i+1}+2^{j+1}\right) B}(x) \\
& \times \sup _{2^{j} r_{B} \leq t<2^{j+1} r_{B}} 2^{-j(n+1)}\left[2^{-i(\epsilon+n / p-n-1)}+2^{-i(2 M-n / 2-1)}\right]|B|^{-1 / p}
\end{aligned}
$$

where $S_{i}(B)$ and $\widetilde{S}_{i}(B)$ are as in the proof of Theorem 1.1. The rest of the proof is similar to that of Theorem 1.1; we omit the details. This finishes the proof of Theorem 1.2.

\section{Further remarks}

In this section, we establish a variant of Theorems 1.1 and 1.2 for the higher order divergence form elliptic operators with complex bounded measurable coefficients and the higher order Schrödinger-type operators.

To this end, we first recall some notion and notations. For $\theta \in[0, \pi)$, the closed sector, $S_{\theta}$, of angle $\theta$ in the complex plane $\mathbb{C}$ is defined by $S_{\theta} \equiv\{z \in \mathbb{C} \backslash\{0\}:|\arg z| \leq \theta\} \cup\{0\}$. Let $\omega \in[0, \pi)$. A closed operator $T$ in $L^{2}\left(\mathbb{R}^{n}\right)$ is called of type $\omega$ (see, for example, [28]), if its spectrum, $\sigma(T)$, is contained in $S_{\omega}$, and for each $\theta \in(\omega, \pi)$, there exists a nonnegative constant $C$ such that for all $z \in \mathbb{C} \backslash S_{\theta},\left\|(T-z I)^{-1}\right\|_{\mathcal{L}\left(L^{2}\left(\mathbb{R}^{n}\right)\right)} \leq C|z|^{-1}$, where and in what follows, $\|S\|_{\mathcal{L}(\mathcal{H})}$ denotes the operator norm of the linear operator $S$ on the normed linear space $\mathcal{H}$. Let $T$ be a one-to-one operator of type $\omega$, with $\omega \in[0, \pi)$ and $\mu \in(\omega, \pi)$, and $f \in H_{\infty}\left(S_{\mu}^{0}\right) \equiv\left\{f\right.$ is holomorphic on $\left.S_{\mu}^{0}:\|f\|_{L^{\infty}\left(S_{\mu}^{0}\right)}<\infty\right\}$, where $S_{\mu}^{0}$ denotes the interior of $S_{\mu}$. By the $H_{\infty}$ functional calculus, the function of the operator $T, f(T)$ is well defined. The operator $T$ is said to have a bounded $H_{\infty}$ functional calculus in the Hilbert space $\mathcal{H}$, if there exist $\mu \in(0, \pi)$ and positive constant $C$ such that for all $\psi \in H_{\infty}\left(S_{\mu}^{0}\right)$, $\|\psi(T)\|_{\mathcal{L}(\mathcal{H})} \leq C\|\psi\|_{L^{\infty}\left(S_{\mu}^{0}\right)}$.

As in [6], let $T$ be an operator defined in $L^{2}\left(\mathbb{R}^{n}\right)$ which satisfies the following assumptions:

$\left(\mathrm{E}_{1}\right)$ The operator $T$ is a one-to-one operator of type $\omega$ in $L^{2}\left(\mathbb{R}^{n}\right)$ with $\omega \in[0, \pi / 2)$;

$\left(\mathrm{E}_{2}\right)$ The operator $T$ has a bounded $H_{\infty}$ functional calculus in $L^{2}\left(\mathbb{R}^{n}\right)$;

$\left(\mathrm{E}_{3}\right)$ Let $k \in \mathbb{N}$. The operator $T$ generates a holomorphic semigroup $\left\{e^{-t T}\right\}_{t>0}$ which satisfies the $k$-Davies-Gaffney estimate, namely, there exist positive constants $C_{6}$ and $C_{7}$ such that for all closed sets $E$ and $F$ in $\mathbb{R}^{n}, t \in(0, \infty)$ and $f \in L^{2}\left(\mathbb{R}^{n}\right)$ supported in $E$,

$$
\left\|e^{-t T} f\right\|_{L^{2}(F)} \leq C_{6} \exp \left\{-\frac{[\operatorname{dist}(E, F)]^{2 k /(2 k-1)}}{C_{7} t^{1 /(2 k-1)}}\right\}\|f\|_{L^{2}(E)} .
$$


When $k=1$, the $k$-Davies-Gaffney estimate is just (2.1).

Let $k \in \mathbb{N}$. Typical examples of operators, satisfying the above assumptions $\left(\mathrm{E}_{1}\right),\left(\mathrm{E}_{2}\right)$ and $\left(\mathrm{E}_{3}\right)$, include the following $2 k$-order divergence form homogeneous elliptic operator

$$
T_{1} \equiv(-1)^{k} \sum_{|\alpha|=|\beta|=k} \partial^{\alpha}\left(a_{\alpha, \beta} \partial^{\beta}\right)
$$

with complex bounded measurable coefficients $\left\{a_{\alpha, \beta}\right\}_{|\alpha|=|\beta|=k}$, and the following $2 k$-order Schrödinger-type operator

$$
T_{2} \equiv(-\Delta)^{k}+V^{k}
$$

with $0 \leq V \in L_{\text {loc }}^{k}\left(\mathbb{R}^{n}\right)$.

For all $f \in L^{2}\left(\mathbb{R}^{n}\right)$ and $x \in \mathbb{R}^{n}$, define the T-adapted square function $S_{T} f(x)$ by

$$
S_{T} f(x) \equiv\left\{\iint_{\Gamma(x)}\left|t^{2 k} T e^{-t^{2 k} T} f(y)\right|^{2} \frac{d y d t}{t^{n+1}}\right\}^{1 / 2} .
$$

Using the $T$-adapted square function $S_{T} f$, Cao and Yang [6] introduced the following Hardy space $H_{T}^{p}\left(\mathbb{R}^{n}\right)$ associated to $T$.

Definition 4.1 ([6]). Let $p \in(0,1]$ and $T$ satisfy the assumptions $\left(\mathrm{E}_{1}\right),\left(\mathrm{E}_{2}\right)$ and $\left(\mathrm{E}_{3}\right)$. A function $f \in L^{2}\left(\mathbb{R}^{n}\right)$ is said to be in $\mathbb{H}_{T}^{p}\left(\mathbb{R}^{n}\right)$ if $S_{T} f \in L^{p}\left(\mathbb{R}^{n}\right)$; moreover, define $\|f\|_{H_{T}^{p}\left(\mathbb{R}^{n}\right)} \equiv\left\|S_{T} f\right\|_{L^{p}\left(\mathbb{R}^{n}\right)}$. The Hardy space $H_{T}^{p}\left(\mathbb{R}^{n}\right)$ is then defined to be the completion of $\mathbb{H}_{T}^{p}\left(\mathbb{R}^{n}\right)$ with respect to the quasi-norm $\|\cdot\|_{H_{T}^{p}\left(\mathbb{R}^{n}\right)}$.

Let $i \in\{1,2\}$. By first establishing the molecular characterization of $H_{T_{i}}^{p}\left(\mathbb{R}^{n}\right)$, Cao and Yang [6] then obtain the following boundedness of the Riesz transform $\nabla^{k}\left(T_{i}^{-1 / 2}\right)$ from $H_{T_{i}}^{p}\left(\mathbb{R}^{n}\right)$ to $H^{p}\left(\mathbb{R}^{n}\right)$ when $p \in(n /(n+k), 1]$.

Theorem 4.1 ([6]). Let $k \in \mathbb{N}, p \in(n /(n+k), 1], T_{1}$ be the $2 k$-order divergence form homogeneous elliptic operator with complex bounded measurable coefficients as in (4.1), and $T_{2}$ the $2 k$-order Schrödinger-type operator as in (4.2). Then, for $i \in\{1,2\}$, the Riesz transform $\nabla^{k}\left(T_{i}^{-1 / 2}\right)$ is bounded from $H_{T_{i}}^{p}\left(\mathbb{R}^{n}\right)$ to $H^{p}\left(\mathbb{R}^{n}\right)$.

Again, for $i \in\{1,2\}$, applying the molecular characterization of $H_{T_{i}}^{p}\left(\mathbb{R}^{n}\right)$ from [6], by an argument similar to that used in the proof of Theorem 1.2, we obtain the endpoint boundedness of $\nabla^{k}\left(T_{i}^{-1 / 2}\right)$ in the critical case that $p=n /(n+k)$. We omit the details by similarity.

Theorem 4.2. Let $k \in \mathbb{N}, p \equiv n /(n+k), T_{1}$ be the $2 k$-order divergence form homogeneous elliptic operator with complex bounded measurable coefficients as in (4.1), and $T_{2}$ the $2 k$ order Schrödinger-type operator as in (4.2). Then, for $i \in\{1,2\}$, the Riesz transform $\nabla^{k}\left(T_{i}^{-1 / 2}\right)$ is bounded from $H_{T_{i}}^{p}\left(\mathbb{R}^{n}\right)$ to $W H^{p}\left(\mathbb{R}^{n}\right)$.

Acknowledgements. The second author is supported by National Natural Science Foundation (Grant No. 11171027) of China and Program for Changjiang Scholars and Innovative Research Team in University of China. 


\section{References}

[1] Ahn, B., Li, J.: Orlicz-Hardy spaces associated to oprators satisfying bounded $H_{\infty}$ functional calculus and Davies-Gaffney estimates. J. Math. Anal. Appl. 373, 485-501 (2011)

[2] Auscher, P., Duong, X.T., McIntosh, A.: Boundedness of Banach space valued singular integral operators and Hardy spaces. Unpublished preprint (2005)

[3] Auscher, P., McIntosh, A., Russ, E.: Hardy spaces of differential forms on Riemannian manifolds. J. Geom. Anal. 18, 192-248 (2008)

[4] Auscher, P., Russ, E.: Hardy spaces and divergence operators on strongly Lipschitz domains of $\mathbb{R}^{n}$. J. Funct. Anal. 201, 148-184 (2003)

[5] Cao, J., Liu, L., Yang, D.: Hardy spaces $H_{\mathcal{L}}^{1}\left(\mathbb{R}^{n}\right)$ associated to Schrödinger type operators $(-\Delta)^{2}+V^{2}$. Houston J. Math. 36, 1067-1095 (2010)

[6] Cao, J., Yang, D.: Hardy spaces $H_{L}^{p}\left(\mathbb{R}^{n}\right)$ associated to operators satisfying $k$-DaviesGaffney estimates. Submitted or arXiv:1107.5365

[7] Coifman, R.R., Weiss, G.: Extensions of Hardy spaces and their use in analysis. Bull. Amer. Math. Soc. 83, 569-645 (1977)

[8] Duong, X.T., Li, J.: Hardy spaces associated to operators satisfying bounded $H_{\infty}$ functional calculus and Davies-Gaffney estimates. Preprint

[9] Duong, X.T., Xiao, J., Yan, L.: Old and new Morrey spaces with heat kernel bounds. J. Fourier Anal. Appl. 13 (2007) 87-111 (2007)

[10] Duong, X.T., Yan, L.: New function spaces of BMO type, the John-Nirenberg inequality, interpolation, and applications. Comm. Pure Appl. Math. 58, 1375-1420 (2005)

[11] Duong, X.T., Yan, L.: Duality of Hardy and BMO spaces associated with operators with heat kernel bounds. J. Amer. Math. Soc. 18, 943-973 (2005)

[12] Dziubański, J., Zienkiewicz, J.: Hardy space $H^{1}$ associated to Schrödinger operator with potential satisfying reverse Hölder inequality. Rev. Mat. Ibero. 15, 279-296 (1999)

[13] Dziubański, J., Zienkiewicz, J.: $H^{p}$ spaces for Schrödinger operators. Fourier analysis and related topics (Bpolhk edlewo, 2000), 45-53, Banach Center Publ. 56, Polish Acad. Sci., Warsaw (2002)

[14] Fefferman, C., Stein, E.M.: $H^{p}$ spaces of several variables. Acta Math. 129, 137-193 (1972)

[15] Fefferman, R., Soria, F.: The space weak $H^{1}$. Studia Math. 85, 1-16 (1986)

[16] Hofmann, S., Lu, G., Mitrea, D., Mitrea, M., and Yan, L.: Hardy spaces associated to non-negative self-adjoint operators satisfying Davies-Gaffney estimates. Mem. Amer. Math. Soc. 214, no. 1007 , vi+78 pp (2011)

[17] Hofmann, S., Martell, J.: $L^{p}$ bounds for Riesz transforms and square roots associated to second order elliptic operators. Publ. Mat. 47, 497-515 (2003)

[18] Hofmann, S., Mayboroda, S.: Hardy and BMO spaces associated to divergence form elliptic operators. Math. Ann. 344, 37-116 (2009)

[19] Hofmann, S., Mayboroda, S.: Correction to "Hardy and BMO spaces associated to divergence form elliptic operators". arXiv: 0907.0129 
[20] Hofmann, S., Mayboroda, S., McIntosh, A.: Second order elliptic operators with complex bounded measurable coefficients in $L^{p}$, Sobolev and Hardy spaces. Ann. Sci. École Norm. Sup. (4) (to appear) or arXiv: 1002.0792

[21] Jiang, R., Yang, D.: Orlicz-Hardy spaces associated with operators satisfying DaviesGaffney estimates. Commun. Contemp. Math. 13, 331-373 (2011)

[22] Jiang, R., Yang, D.: Predual spaces of Banach completions of Orlicz-Hardy spaces associated with operators. J. Fourier Anal. Appl. 17, 1-35 (2011)

[23] Jiang, R., Yang, D.: New Orlicz-Hardy spaces associated with divergence form elliptic operators. J. Funct. Anal. 258, 1167-1224 (2010)

[24] Jiang, R., Yang, D.: Generalized vanishing mean oscillation spaces associated with divergence form elliptic operators. Integral Equations Operator Theory 67, 123-149 (2010)

[25] Jiang, R., Yang, D., Zhou, Y.: Orlicz-Hardy spaces associated with operators. Sci. China Ser. A 52, 1042-1080 (2009)

[26] Liu, H.: The weak $H^{p}$ spaces on homogenous groups. in: Harmonic analysis (Tianjin, 1988), 113-118, Lecture Notes in Math., 1984, Springer, Berlin (1991)

[27] Lu, S.: Four Lectures on Real $H^{p}$ Spaces. World Scientific Publishing Co. Inc., River Edge, NJ (1995)

[28] McIntosh, A.: Operators which have an $H_{\infty}$ functional calculus. Miniconference on operator theory and partial differential equations (North Ryde, 1986), 210-231, Proc. Centre Math. Anal., Austral. Nat. Univ., 14, Austral. Nat. Univ., Canberra (1986)

[29] Quek, T., Yang, D.: Calderón-Zygmund-type operators on weighted weak Hardy spaces over $\mathbb{R}^{n}$. Acta Math. Sin. (Engl. Ser.) 16, 141-160 (2000)

[30] Stein, E.M.: Harmonic Analysis: Real-Variable Methods, Orthogonality, and Oscillatory Integrals. Princeton University Press, Princeton, N. J. (1993)

[31] Stein, E.M., Taibleson, M.H., Weiss, G.: Weak type estimates for maximal operators on certain $H^{p}$ classes. Proceedings of the Seminar on Harmonic Analysis (Pisa, 1980), Rend. Circ. Mat. Palermo 2, 81-97 (1981)

[32] Stein, E.M., Weiss, G.: On the theory of harmonic functions of several variables. I. The theory of $H^{p}$-spaces. Acta Math. 103, 25-62 (1960)

[33] Taibleson, M.H., Weiss, G.: The molecular characterization of certain Hardy spaces, Representation theorems for Hardy spaces. pp. 67-149, Astérisque, 77, Soc. Math. France, Paris (1980)

[34] Trotter, H.F.: On the product of semi-groups of operators, Proc. Amer. Math. Soc. $10,545-551$ (1959)

[35] Yan, L.: Classes of Hardy spaces associated with operators, duality theorem and applications. Trans. Amer. Math. Soc. 360, 4383-4408 (2008)

[36] Yang, Da., Yang, Do., Zhou, Y.: Endpoint properties of localized Riesz transforms and fractional integrals associated to Schrödinger operators. Potential Anal. 30, 271$300(2009)$

[37] Yang, D., Zhou, Y.: Localized Hardy spaces $H^{1}$ related to admissible functions on RD-spaces and applications to Schrödinger operators. Trans. Amer. Math. Soc. 363, 1197-1239 (2011) 